ORLICZ CENTENARY VOLUME

BANACH CENTER PUBLICATIONS, VOLUME 64

INSTITUTE OF MATHEMATICS

POLISH ACADEMY OF SCIENCES

WARSZAWA 2004

\title{
THE REVIVAL OF THE RIEMANNIAN APPROACH TO INTEGRATION
}

\author{
JAROSLAV KURZWEIL \\ Mathematical Institute, Academy of Sciences of the Czech Republic \\ Žitná 25, 11567 Praha 1, Czech Republic \\ E-mail: kurzweil@math.cas.cz
}

Riemannian sums were used in a definition of a nonabsolutely convergent integration on $\mathbb{R}$ almost fifty years ago and it was prooved that the new integration is equivalent to Perron integration. Therefore it looked like there appeared just a new approach to integration. But the Riemannian approach proved to be very flexible: (i) it is the basis of numerous integrations of real functions of a real variable, (ii) it opens new ways in integration of functions of several variables, (iii) it is well applicable to integration of vector valued functions, (iv) it admits a great degree of abstraction. This paper is concentrated on (i) with a special accent on structures (convergence, topology) on the space of integrable functions. Section 5 contains a brief information concerning (ii).

1. Preliminaries. Let $I=[a, b] \subset \mathbb{R}$ and let $|E|$ denote the Lebesgue measure of $E \subset I$. A set $A \subset I$ is a figure if it is a finite union of compact intervals.

A partition in $I$ is a set $\Delta=\left\{\left(t_{1}, A_{1}\right), \ldots,\left(t_{k}, A_{k}\right)\right\}$ where $t_{i} \in I, A_{i}$ is a figure and $\left|A_{i} \cap A_{j}\right|=0$ for $i \neq j$. If in addition $\bigcup A_{i}=I$ then $\Delta$ is a partition of $I$. Let $\xi: I \rightarrow(0, \infty)$. Then $\Delta$ is called $\xi$-fine if $A_{i} \subset\left(t_{i}-\xi\left(t_{i}\right), t_{i}+\xi\left(t_{i}\right)\right)$ for $i=1,2, \ldots, k$.

If $H: I \rightarrow \mathbb{R}, A=\left[c_{1}, d_{1}\right] \cup \cdots \cup\left[c_{k}, d_{k}\right]$ where $a \leq c_{1}<d_{1} \leq \cdots \leq c_{k}<d_{k} \leq b$, we put

$$
H(A)=\sum_{j=1}^{k}\left(H\left(d_{j}\right)-H\left(c_{j}\right)\right) .
$$

2. $\mathcal{Y}$-integration. Let $\mathcal{L}$ be the set of partitions in $I$, and let $\mathcal{H} \mathcal{K}$ be the set of partitions $\theta=\left\{\left(s_{1}, J_{1}\right), \ldots,\left(s_{l}, J_{l}\right)\right\}$ in $I$ such that $s_{m} \in J_{m}$ and $J_{m}$ is an interval for $m=1,2, \ldots, l$. A set $\mathcal{Y}$ of partitions $\Delta$ in $I$ is called an integration base if $\mathcal{H} \mathcal{K} \subset \mathcal{Y}$, if $\Delta \in \mathcal{Y}$ and $\Psi \subset \Delta$ implies $\Psi \in \mathcal{Y}$ and if $\Delta \cup \theta \in \mathcal{Y}$ for $\Delta \in \mathcal{Y}, \theta \in \mathcal{H K}$ where $\left|A_{i} \cap J_{m}\right|=0$ for all $i, m$.

2000 Mathematics Subject Classification: Primary 26A39.

The paper is in final form and no version of it will be published elsewhere. 
2.1. Definition. Let $f: I \rightarrow \mathbb{R}, w \in \mathbb{R}$. Then $f$ is called $\mathcal{Y}$-integrable on $I$ and $w$ is called the $\mathcal{Y}$-integral of $f$ over $I\left(w=(\mathcal{Y}) \int_{I} f \mathrm{~d} t\right)$ if there exists $\delta: \mathbb{N} \times I \rightarrow(0, \infty)$ such that

$$
\left|w-\sum_{i} f\left(t_{i}\right)\right| A_{i}|| \leq 2^{-j} \quad \text { for } j \in \mathbb{N}, \Delta \in \mathcal{Y}
$$

$\Delta$ being a $\delta(j, \cdot)$-fine partition of $I$.

2.2. Note. Definition 2.1 with $\mathcal{Y}=\mathcal{H} \mathcal{K}$ was introduced in [Kur 57] and it was proved that $\mathcal{H} \mathcal{K}$-integration is Perron integration (see also [He 61], [He 63]). The motivation came from a convergence problem in the theory of ordinary differential equations (see [Kur 57] or [Kur]). Riemannian approach to Lebesgue integration appeared in [Mc Sh 69], other integrations were introduced in [Bo-Pf 92] and [Bo 96]. For a survey of results on the Riemannian approach to integration see also [Bo 03].

\subsection{Elementary properties}

(2.1) $\quad w$ is unique since for every $\xi: I \rightarrow(0, \infty)$ there exists a $\xi$-fine partition $\Delta$ of $I, \Delta \in \mathcal{H} \mathcal{K}$.

(2.2) As a direct consequence of $\mathcal{H} \mathcal{K} \subset \mathcal{Y}$ we get: if $f$ is $\mathcal{Y}$-integrable over $I$, then $f$ is $\mathcal{H} \mathcal{K}$-integrable over $I$ and $(\mathcal{Y}) \int_{I} f \mathrm{~d} t=(\mathcal{H} \mathcal{K}) \int_{I} f \mathrm{~d} t$.

(2.3) If $f$ is $\mathcal{Y}$-integrable over $I$ then it is $\mathcal{Y}$-integrable over every subinterval of $I$.

(2.4) Let $f$ be $\mathcal{Y}$-integrable over $I$. Set $F(a)=0, F(s)=(\mathcal{Y}) \int_{[a, s]} f \mathrm{~d} t$ for $a<s \leq b$; $F$ is called the primitive of $f$.

$F$ is continuous and differentiable a.e., $\dot{F}=f$ a.e.

$P_{\mathcal{Y}}$ is the set of primitives of functions which are $\mathcal{Y}$-integrable over $I$.

(2.5) $\quad P_{\mathcal{L}} \subset P_{\mathcal{Y}} \subset P_{\mathcal{H} K}($ since $\mathcal{H} \mathcal{K} \subset \mathcal{Y} \subset \mathcal{L})$.

(2.6) $\quad P_{\mathcal{L}}$ is the set of absolutely continuous functions $F: I \rightarrow \mathbb{R}, F(a)=0$ (cf. [Mc Sh 69]).

$P_{\mathcal{H} K}$ is the set of $\mathrm{ACG}_{*}$-functions (since Perron integration is equivalent to Denjoy integration in the restricted sense, cf. [Sa 37]).

The emphasis will be put on the concept of the primitive (in order to avoid classes of equivalent functions).

2.4. Remark. A fundamentally different characterization of $P_{\mathcal{H} K}$ was given in [B-P-S 95]:

Let $F: I \rightarrow \mathbb{R}, F(a)=0$. Then $F \in P_{\mathcal{H} \mathcal{K}}$ if and only if $F$ generates an absolutely continuous variational measure. See also [Pf 99].

3. Convergence in $P_{\mathcal{Y}}$. Let $\delta: \mathbb{N} \times I \rightarrow(0, \infty), \xi: I \rightarrow(0, \infty)$. By $Q^{*}(\mathcal{Y}, \delta, \xi)$ denote the set of $F: I \rightarrow \mathbb{R}$ such that there exist $f: I \rightarrow \mathbb{R}$ and $N \subset I,|N|=0$ fulfilling

$$
\sum_{i}\left|F\left(A_{i}\right)\right| \leq 2^{-j}+\sum_{i} \xi\left(t_{i}\right)\left|A_{i}\right|
$$

for $j \in \mathbb{N}$ and every $\delta(j, \cdot)$-fine $\Delta=\left\{\left(t_{i}, A_{i}\right)\right\} \in \mathcal{Y}$,

$$
\sum_{i}\left|F\left(A_{i}\right)-f\left(t_{i}\right)\right| A_{i}|| \leq 2^{-j}
$$


for $j \in \mathbb{N}$ and every $\delta(j, \cdot)$-fine $\Delta=\left\{\left(t_{i}, A_{i}\right)\right\} \in \mathcal{Y}$ where $t_{i} \in I \backslash N$ for all $i$. Then

$$
P_{\mathcal{Y}}=\bigcup_{\delta, \xi} Q^{*}(\mathcal{Y}, \delta, \xi),
$$

if $F_{p} \in Q^{*}(\mathcal{Y}, \delta, \xi)$ f or $p \in \mathbb{N}$ and if $F: I \rightarrow \mathbb{R}$ is bounded, $\left\|F_{p}-F\right\|_{\text {sup }} \rightarrow 0$ for $p \rightarrow \infty$ then $F \in P_{\mathcal{Y}}$ and $f_{p} \rightarrow f$ in measure for $p \rightarrow \infty$, where $F_{p}$ and $F$ are the primitives of $f_{p}, f$.

The definition of $Q^{*}(\mathcal{Y}, \delta, \xi)$ differs only slightly from the definition of $Q_{\mathcal{Y}}(\delta)$ in [Kur 02], Definition 2.6. The proofs of (3.3) and (3.4) are analogous to the proofs in [Kur 02], Theorems 2.9 and 2.10. (3.3) may be interpreted as an equivalent definition of $P \mathcal{Y}$ and (3.4) implies that $Q^{*}(\mathcal{Y}, \delta, \xi)$ is closed in the space of continuous functions; in fact, it is compact.

3.1. Definition. Let $F_{p} \in P_{\mathcal{Y}}$ for $p \in \mathbb{N}, F \in P_{\mathcal{Y}}$. The sequence $F_{p}$ is called $Q_{y^{-}}^{*}$ convergent to $F, F_{p} \stackrel{\mathbb{Q}_{y}^{*}}{\longrightarrow} F$, if there exist $\delta$ and $\xi$ such that

$$
F_{p} \in Q^{*}(\mathcal{Y}, \delta, \xi) \quad \text { for } p \in \mathbb{N}
$$

and

$$
\left\|F_{p}-F\right\|_{\text {sup }} \rightarrow 0 \quad \text { for } p \rightarrow \infty .
$$

$Q_{y}^{*}$-convergence resembles the two-norm convergence (cf. [Al 50], [W 61]).

3.2. Remark. Convergence on $P_{\mathcal{Y}}$ can be introduced in another way. Call the sequence $F_{p} \mathbb{E}_{y}$-convergent to $F, F_{p} \stackrel{\mathbb{E}_{y}}{\longrightarrow} F$, if there exist $f_{p}, f: I \rightarrow \mathbb{R}$ such that

(3.7) there exists $\delta: \mathbb{N} \times I \rightarrow(0, \infty)$ such that

$$
\left|F_{p}(I)-\sum_{i} f_{p}\left(t_{i}\right)\right| A_{i}|| \leq 2^{-j}
$$

for $p, j \in \mathbb{N}, \Delta=\left\{\left(t_{i}, A_{i}\right)\right\} \in \mathcal{Y}, \Delta$ being a $\delta(j, \cdot)$-fine partition of $I$; $f_{p}(t) \rightarrow f(t)$ for $p \rightarrow \infty, t \in I$.

3.3. Remark. $F_{p} \stackrel{\mathbb{E}_{y}}{\longrightarrow} F$ implies that $F_{p} \stackrel{\mathbb{Q}_{y}^{*}}{\longrightarrow} F$; on the other hand if $F_{p} \stackrel{\mathbb{Q}_{y}^{*}}{\longrightarrow} F$ then there exists a subsequence $q(p)$ such that $F_{q(p)} \stackrel{\mathbb{E}_{y}}{\longrightarrow} F$.

3.4. Remark. $F: I \rightarrow \mathbb{R}$ is an $A C G_{*}$-function if there exists a sequence of sets $E_{i} \subset I$ such that $F$ in relation to each $E_{i}$ has a property which resembles absolute continuity. In this way a descriptive characterization of functions from $P_{\mathcal{H} K}$ is obtained. The concept of controlled convergence has the same roots (cf. [D 51], [Ch-D 78], [L 89]). In general it can be said that controlled convergence is equivalent to $\mathbb{E}_{\mathcal{H} \mathcal{K}}$-convergence. For a general approach see [Bo-Pi 91]. There is treated a more general case of integration of $f$ with respect to a function $G: I \rightarrow \mathbb{R}$, the most important results being valid if $G \in P_{\mathcal{H} \mathcal{K}}$. See also [G 91] and [G 92].

4. Topologization of $P_{\mathcal{Y}}$. Let $\mathcal{T}(\mathcal{Y})$ be the set of locally convex topologies $\tau$ on $P_{\mathcal{Y}}$ such that

$$
F_{p} \stackrel{\mathbb{Q}_{y}^{*}}{\longrightarrow} F \text { implies } F_{p} \rightarrow F \text { in }\left(P_{\mathcal{Y}}, \tau\right)
$$


Since $\mathcal{T}(\mathcal{Y}) \neq \emptyset$ there exists a unique locally convex topology $\mathcal{U}_{L C}\left(\mathbb{Q}_{y}^{*}\right) \in \mathcal{T}(\mathcal{Y})$ which is finer than any topology $\tau \in \mathcal{T}(\mathcal{Y})$. If $\mathcal{U}_{L C}\left(\mathbb{E}_{y}\right)$ has an analogous meaning then $\mathcal{U}_{L C}\left(\mathbb{E}_{y}\right)=$ $\mathcal{U}_{L C}\left(\mathbb{Q}_{y}^{*}\right)$, cf. Remark 3.3.

4.1. Theorem. $\mathcal{U}_{L C}\left(\mathbb{E}_{\mathcal{L}}\right)$ is the topology induced on $P_{\mathcal{L}}$ by the norm $\|\cdot\|_{\text {var }}$ where $\|F\|_{\text {var }}=\operatorname{var} F$ (cf. [Kur 02], Theorems 5.2 and 5.3).

4.2. Theorem. $\mathcal{U}_{L C}\left(\mathbb{E}_{\mathcal{H} \mathcal{K}}\right)$ is the topology induced on $P_{\mathcal{H} K}$ by the norm $\|\cdot\|_{\text {sup }}$ (cf. [Kur 02], Theorem 8.2 and Note 8.1 (ii)).

Observe that $\left(P_{\mathcal{L}}, \mathcal{U}_{L C}\left(\mathbb{E}_{\mathcal{L}}\right)\right)$ is complete and $\left(P_{\mathcal{H} \mathcal{K}}, \mathcal{U}_{L C}\left(\mathbb{E}_{\mathcal{H} \mathcal{K}}\right)\right)$ is not complete. It is desirable to get sufficient conditions for the completeness or noncompleteness of $\left(P_{\mathcal{Y}}, \mathcal{U}_{L C}\left(\mathbb{E}_{y}\right)\right)$. A theoretical approach to this problem was obtained in [Kur 02], Chapters 4 and 7 for a rather wide class of integration bases $\mathcal{Y}$ but only some concrete results will be described here.

4.3. Notation. Let $\Lambda$ be the set of $\lambda:[0, \infty) \rightarrow[0, \infty)$ nondecreasing, $\lambda(\sigma)>0$ for $\sigma>0$. Denote by $\operatorname{dist}(t, E)$ the distance of $t$ from $E$ where $t \in I, E \subset I$.

For $\lambda \in \Lambda$ let $\mathcal{S}(\lambda)$ be the set of partitions $\Delta=\left\{\left(t_{i}, A_{i}\right)\right\} \in \mathcal{L}$ such that

$$
\begin{gathered}
\text { if }\left(t_{i}, A_{i}\right) \in \Delta \text { then } A_{i} \text { is an interval, } \\
\qquad \sum_{i} \lambda\left(\operatorname{dist}\left(t_{i}, A_{i}\right)\right) \leq 1 .
\end{gathered}
$$

4.4. TheOrem. Let $\lambda \in \Lambda$. Then $\left(P_{\mathcal{S}(\lambda)}, \mathcal{U}_{L C}\left(\mathbb{E}_{\mathcal{S}(\lambda)}\right)\right.$ is not complete (cf. Remark 3.2 and [Kur 02], Theorem 8.11).

4.5. Remark. Put $\lambda(i, \sigma)=2^{-i} \sigma$ for $\sigma \geq 0, i \in \mathbb{N}$. In [Bo 96], $*$-integration was defined. It is a direct consequence of the definition that $F$ is the primitive of a $*$-integrable $f$ if and only if

$$
F \in \bigcap_{i} P_{\mathcal{S}(\lambda(i, \cdot))}
$$

Moreover, $F$ is the primitive of a $*$-integrable $f$ if and only if there exist an everywhere differentiable $G$ and an absolutely continuous $H$ such that

$$
F=G+H
$$

(cf. [B-P-P 00]).

Let $\lambda, \eta \in \Lambda, \lambda(\sigma) / \eta(\sigma) \rightarrow 0$ for $\sigma \rightarrow 0$. Then

$$
\begin{aligned}
& P_{\mathcal{S}(\eta)} \subset P_{\mathcal{S}(\lambda)}, P_{\mathcal{S}(\lambda)} \backslash P_{\mathcal{S}(\eta)} \neq \emptyset \text { and } \mathcal{U}_{L C}\left(\mathbb{E}_{\mathcal{S}(\eta)}\right) \text { is finer than the restriction } \\
& \left.\mathcal{U}_{L C}\left(\mathbb{E}_{\mathcal{S}(\lambda)}\right)\right|_{P_{\mathcal{S}}(\eta)}
\end{aligned}
$$

(cf. [Kur 02], Theorem 8.11). It can also be proved that

$$
\bigcap_{\lambda \in \Lambda} P_{\mathcal{S}(\lambda)}=P_{\mathcal{L}}
$$

Therefore $P_{\mathcal{S}(\lambda)}$ seems to be rather close to $P_{\mathcal{L}}$ if $\lambda \rightarrow 0$ rapidly but the results on completeness are different. 
4.6. Notation. The regularity of a couple $(t, A)$ where $t \in I$ and $A$ is a figure is defined by

$$
\operatorname{reg}(t, A)=\frac{|A|}{\# \partial A \cdot \operatorname{diam}(\{t\} \cup A)}
$$

where $\# \partial A$ is the number of boundary points of $A$ and diam $E$ is the diameter of $E$. (We have $0<\operatorname{reg}(t, A) \leq \frac{1}{2}$ for every couple $(t, A)$.)

Let $\Omega$ be the set of nondecreasing functions $\omega:(0, \infty) \rightarrow\left(0, \frac{1}{2}\right]$. For $\omega \in \Omega$ let $\mathcal{R}(\omega)$ be the set of partitions $\Delta \in \mathcal{L}$ such that

$$
\operatorname{reg}(t, A) \geq \omega(\operatorname{diam}(\{t\} \cup A)) \quad \text { for } \quad(t, A) \in \Delta
$$

and let $\mathcal{R}(\omega, 1)$ be the set of partitions $\Delta \in \mathcal{R}(\omega)$ such that

$$
t \in A \text { for }(t, A) \in \Delta .
$$

4.7. Theorem. Neither $\left(P_{\mathcal{R}(\omega)}, \mathcal{U}_{L C}\left(\mathbb{E}_{\mathcal{R}(\omega)}\right)\right)$ nor $\left(P_{\mathcal{R}(\omega, 1)}, \mathcal{U}_{L C}\left(\mathbb{E}_{\mathcal{R}(\omega, 1)}\right)\right)$ is complete (cf. [Kur 02], Theorem 9.2, Lemma 9.4, Note 9.5).

4.8. Remark. $\left(P_{\mathcal{H} \mathcal{K}}, \mathcal{U}_{L C}\left(\mathbb{E}_{\mathcal{H} \mathcal{K}}\right)\right)$ is not complete. On the other hand there exists a topology $\tau$ on $P_{\mathcal{H} \mathcal{K}}$ such that

$$
\left(P_{\mathcal{H} \mathcal{K}}, \tau\right) \text { is a complete topological vector space, }
$$

$$
\text { if } F_{p} \stackrel{\mathbb{E}_{H K}}{\longrightarrow} F \text { then } F_{p} \rightarrow F \text { in }\left(P_{\mathcal{H} \mathcal{K}}, \tau\right)
$$

(cf. (2.6) and [Kur 00], Theorem 8.1).

4.9. REMARK. Let $P^{\Delta}$ be the set of primitives with respect to the integration on onedimensional intervals which was introduced in [Bo-Pf 92]. We have

$$
P^{\Delta}=\bigcap_{j} P_{\mathcal{R}\left(\omega_{j}, 1\right)}
$$

where $\omega_{j}(\sigma)=2^{-j}$ for $j \in \mathbb{N}, \sigma>0$.

Let $F_{p}, F \in P^{\Delta}$ for $p \in \mathbb{N}$. The sequence $F_{p}$ is called $\mathbb{E}^{\Delta}$-convergent to $F, F_{p} \stackrel{\mathbb{E}^{\Delta}}{\longrightarrow} F$, if $F_{p} \stackrel{\mathbb{E}_{\mathcal{R}\left(\omega_{j}, 1\right)}}{\longrightarrow} F$ for every $j \in \mathbb{N}$. The $\mathcal{U}_{L C}\left(\mathbb{E}^{\Delta}\right)$ is defined in the same way as $\mathcal{U}_{L C}\left(\mathbb{E}_{y}\right)$ in Theorem 3.1. It can be proved by methods from [Kur 02], Ch. 10 that $\left(P^{\Delta}, \mathcal{U}_{L C}\left(\mathbb{E}^{\Delta}\right)\right)$ is not complete (this result may differ from Theorem 10.6, Remark 1 [Kur 02] since the topologies $\mathcal{U}_{L C}\left(\mathbb{E}^{\Delta}\right)$ and $\mathcal{U}_{\chi\left(\mathcal{R}^{*}(1)\right)}$ from the theorem mentioned above need not coincide).

Theorems 4.4 and 4.7 evoke the following question: Does there exist an integration base $\mathcal{Y}$ such that $P_{\mathcal{Y}} \neq P_{\mathcal{L}}$ and $\left(P_{\mathcal{Y}}, \mathcal{U}_{L C}\left(\mathbb{Q}_{y}^{*}\right)\right)$ is complete?

The answer is affirmative.

Let $\mathcal{M}(\{a\})$ be the set of partitions $\Delta=\left\{\left(t_{i}, A_{i}\right)\right\} \in \mathcal{L}$ such that

$$
\text { if } t_{i}=a \text { then } A_{i}=[a, s], a<s \leq b .
$$

4.10. TheOrem. $P_{\mathcal{M}(\{a\})}$ is the set of $F: I \rightarrow \mathbb{R}, F(a)=0$ such that $F$ is continuous and the restriction $\left.F\right|_{[s, b]}$ is absolutely continuous for $s \in(a, b)$. The topology $\mathcal{U}_{L C}\left(\mathbb{E}_{\mathcal{M}\{(a\})}\right)$ is induced by the set of seminorms $\left\{\|\cdot\|_{\text {sup }},\|\cdot\|_{s}^{*} ; s \in(a, b)\right\}$ where $\|F\|_{s}^{*}$ is the variation of $F$ on $[s, b]$. Moreover, $\left(P_{\mathcal{M}(\{a\})}, \mathcal{U}_{L C}\left(\mathbb{E}_{\mathcal{M}(\{a\})}\right)\right)$ is complete. 
4.11. Remark. Let $S \subset I$ be countable and closed. $\mathcal{M}(S)$ can be defined analogously to $\mathcal{M}(\{a\})$ and the above result can be extended to the integration base $\mathcal{M}(S)$ (cf. [Kur 00], Chapter 6).

4.12. REMARK. Nonabsolute integration was approached by the method of truncation (cf. [Ti 29], [O 54], [U 56], [A-A 65]). For the concept of a ranked space and its application to nonabsolute integration see [Kun 54], [Kun 56], [N 78,79], [N 90] and for numerous results which include the completion of st with respect to a quasinorm and the ranked completion of st see [N 01]. Here st is the set of step functions.

5. A short excursion to integration in $\mathbb{R}^{k}$. $\mathcal{H} \mathcal{K}$-integration in $\mathbb{R}^{1}$ has the following two properties:

(5.1) If $F$ is differentiable at every $t \in I$ then

$$
(\mathcal{H} \mathcal{K}) \int_{I} \dot{F} \mathrm{~d} t=F(b)-F(a)
$$

(5.2) The classical transformation formula is valid for diffeomorphisms.

Thus $\mathcal{H} \mathcal{K}$-integration on intervals of dimension 1 can be transferred to integration on curves.

Let now $k>1$,

$$
I=\left[a_{1}, b_{1}\right] \times \cdots \times\left[a_{k}, b_{k}\right], \quad J=\left[c_{1}, d_{1}\right] \times \cdots \times\left[c_{k}, d_{k}\right]
$$

where $\left.a_{i} \leq c_{i}<d_{i} \leq b_{i}, i=1,2, \ldots k\right)$,

$$
\operatorname{reg} J=\frac{\min \left\{d_{i}-c_{i} ; i=1,2, \ldots, k\right\}}{\max \left\{d_{i}-c_{i} ; i=1,2, \ldots, k\right\}} .
$$

(A) If in the definition of the $\mathcal{H} \mathcal{K}$-integral $I$ and $J$ are interpreted as in (5.3) then the resulting integration is equivalent to Perron integration (cf. e.g. [Kur 80]). An analogue of the Fubini theorem is valid but neither (5.1) or (5.2) extend to this integration.

(B) If $0<\alpha<1$ and if the assumption

$$
\operatorname{reg} J \geq \alpha \quad(\text { for all } J \text { which are used in the partitions of } I \text { ) }
$$

is added then the analogue of the Fubini theorem gets lost (cf. [J-K-S 83]) but on the other hand we have

$$
\text { If } F: I \rightarrow \mathbb{R}^{k} \text { is a vector field which is differentiable at every } t \in I \text { then }
$$

$$
\begin{aligned}
& \int_{I} \operatorname{div} F \mathrm{~d} \lambda_{k} \text { exists and } \\
& \int_{I} \operatorname{div} F \mathrm{~d} \lambda_{k}=\int_{\partial I} F \cdot n \mathrm{~d} \lambda_{k-1}
\end{aligned}
$$

where $\lambda_{k}$ is the Lebesgue measure on $\mathbb{R}^{k}$ and $\lambda_{k-1}$ is the Lebesgue measure on $(k-1)$ dimensional hyperplanes, $n$ is the exterior normal to $\partial I$ and F.n denotes the scalar product (cf. [Ma 81]). (5.6) is an obvious extension of (5.1) and the formula in (5.6) is a strange version of the Stokes formula since $\operatorname{div} F$ is general but the domain of integration is very special. 
(C) A satisfactory solution in this situation is due to B. Bongiorno and W. Pfeffer (cf. [Bo-Pf 92]). Without going into details one can say that the role of the intervals is played by $B V$-sets (which are closed sets $A$ in $\mathbb{R}^{k}$ such that the $(m-1)$-dimensional Hausdorff outer measure of the essential boundary of $A$ is finite). In this interpretation of $I$ the formula from (5.6) is valid and (5.2) also extends (cf. also [Pf 93], Ch. 13).

6. S-integration; local systems. S-integration is defined for some maps $\mathbf{S}$ from $\mathbb{R}$ to the set of subsets of $\mathbb{R}$. A very brief information on $\mathbf{S}$-integration is given in this section.

Assume that for every $x \in \mathbb{R}$

$$
\begin{aligned}
& \mathbf{S}(x) \text { is a filter on } \mathbb{R}, \\
& \{x\} \notin \mathbf{S}(x), \\
& \text { if } s \in \mathbf{S}(x) \text { then } x \in s, \\
& \text { if } s \in \mathbf{S}(x) \text { and } \sigma>0 \text { then } s \cap(x-\sigma, x+\sigma) \in \mathbf{S}(x) \text {. }
\end{aligned}
$$

In the terminology of [Bo-Pi-Sk 2002] $\mathbf{S}$ is called a filtering local system.

Let $I=[a, b] \subset \mathbb{R}$. A function $\gamma: I \rightarrow 2^{\mathbb{R}}$ with $\gamma(x) \in \mathbf{S}(x)$ is called an S-choice.

With each choice $\gamma$ we associate the family

$$
\beta_{\gamma}=\{(x,[u, v]) ; x=u, v \in \gamma(x) \text { or } x=v, u \in \gamma(x) \text { where } x \in I\} .
$$

A partition $\Delta=\left\{(x, A\}\right.$ of $I$ is called a $\beta_{\gamma}$-partition of $I$ if $\Delta \subset \beta_{\gamma}$.

Assume in addition that

(6.6) $\mathbf{S}$ is bilateral (i.e. every $s \in \mathbf{S}(x)$ contains points on either side of $x$ ),

(6.7) S satisfies the intersection condition (i.e. for every choice $\gamma$ there exists a positive function $\delta$ such that $0<y-x<\min \{\delta(x), \delta(y)\}$ implies $\gamma(x) \cap \gamma(y) \cap[x, y] \neq \emptyset)$.

Then

(6.8) for any choice $\gamma$ there exists a $\beta_{\gamma}$-partition of $I$.

6.1. Definition. Let $f: I \rightarrow \mathbb{R}, w \in \mathbb{R}$. If for every $\varepsilon>0$ there exists an S-choice $\gamma$ such that

$$
\left|w-\sum_{\Delta} f(x)\right| J|| \leq \varepsilon
$$

for every $\beta_{\gamma}$-partition $\Delta=\{(x, J)\}$ of $I$ then $f$ is $\mathbf{S}$-integrable with the integral $w, w=$ (S) $\int_{I} f \mathrm{~d} t$.

The $\mathbf{S}$-integral has some of the usual elementary properties: (i) If $f$ is $\mathbf{S}$-integrable on $I$ then $f$ is $\mathbf{S}$-integrable on any subinterval of $I ; F$ defined by $F(s)=(\mathbf{S}) \int_{[a, s]} f \mathrm{~d} t$ is called the $\mathbf{S}$-primitive of $f$. (ii) $F$ is $\mathbf{S}$-continuous and the $\mathbf{S}$-derivative of $F$ exists and is equal to $f$ a.e.

$\mathbf{S}$-primitives were examined in connection with a new concept of $A C G$-functions (these functions need not be continuous), $\mathbf{S}$-derivatives, approximate derivatives, $\mathbf{S} A C G$ functions, variational measures (see [B-P-S 02], [E 95]). Further results were obtained in the case that $\mathbf{S}$ is generated by a path system or if $\mathbf{S}$ is a weak path system or if $\mathbf{S}$ is a $p$-adic 
path system or a dyadic path system (see [Th 90], [Th 82/83a, Th 82/83b], [B-P-S 02], [B-P-S 03]).

7. $\mathbf{N}$-integration. $\mathcal{H} \mathcal{K}$-integration can be applied to $\int_{I} f \mathrm{~d} h$ where $f, h: I \rightarrow \mathbb{R}$ in an obvious way. If $h$ is continuous then $\mathbf{N}$-integration and $\mathcal{H} \mathcal{K}$-integration are equivalent. For some discontinuous $h$ the $\mathbf{N}$-integration is a true extension of the $\mathcal{H} \mathcal{K}$-integration.

Discontinuous processes are caused by friction, hysteresis, plasticity, action of regulators etc. In terms of mathematics discontinuities appear if we have to do with variational inequalities, the play operator and limit cases of differential equations with a small parameter at derivatives. There is an increasing interest of mathematicians in these problems (cf. [Kr-L 02], [Br-Kr 03]). The concept of regulated functions is a useful tool in this direction.

$g:[a, b] \rightarrow \mathbb{R}$ is called a regulated function on $[a, b]$ if the following limits exist:

$$
\begin{aligned}
& g(t+)=\lim _{s \rightarrow t+} g(s) \quad \text { for } a \leq t<b, \\
& g(t-)=\lim _{s \rightarrow t-} g(s) \quad \text { for } a<t \leq b ;
\end{aligned}
$$

$G(a, b)$ is the space of regulated functions on $[a, b]$.

$G(a, b)$ is usually endowed with the supremum norm. For results on linear functionals, operators and integral equations see e.g. [Ho 75], [Sch 92a, Sch 92b], [Tv 96], [Tv 98].

Let $f, g: I \rightarrow \mathbb{R}$,

$$
g(t)=0 \quad \text { for } t \in I \backslash M, M \text { countable. }
$$

It is well known that the Young integral $(Y) \int_{I} f \mathrm{~d} g$ exists for any $f: I \rightarrow \mathbb{R}$ and, moreover,

$$
(Y) \int_{[a, b]} f \mathrm{~d} g=f(b) g(b)-f(a) g(a) .
$$

However, $(\mathcal{H} \mathcal{K}) \int_{I} f \mathrm{~d} g$ need not exist even if $f$ is continuous. An example of such $f$ and $g$ was based on the bad properties of integral sums

$$
\sum_{i=1}^{k} f\left(t_{i}\right)\left[g\left(x_{i}\right)-g\left(x_{i-1}\right)\right]
$$

where $a=x_{0}<x_{1}<\cdots<x_{k}=b, t_{i} \in\left[x_{i-1}, x_{i}\right]$ for $i=1,2, \ldots, k$ and $x_{i} \in M$ for a large number of $i$ 's (cf. [Kr-Kur 02]).

This situation gave an impulse to an extension of the concept of $(\mathcal{H} \mathcal{K}) \int_{I} f \mathrm{~d} g$ (by avoiding the possibility that $x_{i} \in M$ for any $i=1,2, \ldots, k-1$ ).

Let $\mathbf{N}$ be a system of subsets of $I$ fulfilling

$$
\operatorname{cl}(I \backslash E)=I \quad \text { for } E \in \mathbf{N},
$$

if $E, C \in \mathbf{N}$ then $E \cup C \in \mathbf{N}$.

7.1. Definition. Let $\mathbf{N}$ fulfil (7.6) and (7.7), $\xi: I \rightarrow(0, \infty), E \in \mathbf{N}$. Let $\Delta=$ $\left\{\left(t_{i},\left[x_{i-1}, x_{i}\right]\right) ; i=1,2, \ldots, k\right\} \in \mathcal{H} \mathcal{K}, a=x_{0}<x_{1}<\cdots<x_{k}=b$. 
$\Delta$ is called $(\xi, E)$-fine if

$$
\begin{gathered}
\Delta \text { is } \xi \text {-fine, } \\
x_{i} \in I \backslash E \quad \text { for } i=1,2, \ldots, k-1, \\
t_{i} \in\left(x_{i-1}, x_{i}\right) \quad \text { for } i=2,3, \ldots, k-1 .
\end{gathered}
$$

7.2. Definition. Let $\mathbf{N}$ fulfil (7.5) and (7.6), $f, g: I \rightarrow \mathbb{R}, w \in \mathbb{R} . w$ is called the $\mathbf{N}$-integral over $I$ of $f$ with respect to $g, w=(\mathbf{N}) \int_{I} f \mathrm{~d} g$, if for every $\varepsilon>0$ there exist $\xi: I \rightarrow(0, \infty)$ and $E \in \mathbf{N}$ such that

$$
\left|w-\sum_{i} f\left(t_{i}\right)\left(g\left(x_{i}\right)-g\left(x_{i-1}\right)\right)\right| \leq \varepsilon
$$

for every $(\xi, E)$-fine partition

$$
\Delta=\left\{\left(t_{i},\left[x_{i-1}, x_{i}\right]\right) ; i=1,2, \ldots, k\right\}
$$

of $I$. ( $w$ is unique since for every $(\xi, E))$ there exists a $(\xi, E)$-fine partition $\Delta$ of $I$.)

7.3. Theorem. Let $\mathbf{N}$ fulfil (7.5) and (7.6). Let $f, g: I \rightarrow \mathbb{R}, g$ continuous. If ( $\mathbf{N})$ $\int_{I} f \mathrm{~d} g$ exists, then $(\mathcal{H K}) \int_{I} f \mathrm{~d} g$ exists and both integrals are equal (cf. [Kr 03]; Krejčí writes $K N$-integration and $(K N) \int_{I} f \mathrm{~d} g$ instead of $\mathbf{N}$-integration and $\left.(\mathbf{N}) \int_{I} f \mathrm{~d} g\right)$.

7.4. TheOREM. Let $\mathbf{N}$ be the system of countable subsets of $I, f: I \rightarrow \mathbb{R}, g \in G(a, b)$. If $(Y) \int_{I} f \mathrm{~d} g$ exists then $(\mathbf{N}) \int_{I} f \mathrm{~d} g$ exists and both integrals are equal.

7.5. REMARK. Let $I v$ be the set of nonempty compact subintervals of $I=[a, b]$. A general scheme for Riemannian integration scheme was formulated in [Th 82/83b].

Let $B=\{\beta\}$ be a filter base on $I \times I v$ which has the partitioning property (i.e. for every $\beta \in B$ and every $K \in I v$ there exists a partition $\Delta=\{(t, J)\}$ of $K$ such that $\Delta \subset \beta$.

Let $f: I \rightarrow \mathbb{R}, G: I \rightarrow \mathbb{R}, w \in \mathbb{R} . f$ is called $B$-integrable on $I$ with respect to $G$ and $w$ is the $B$-integral, $w=(B) \int_{I} f \mathrm{~d} g$, if for every $\varepsilon>0$ there exists $\beta \in B$ such that

$$
\left|w-\sum_{\Delta} f(t) G(J)\right| \leq \varepsilon
$$

for every partition $\Delta$ of $I, \Delta \subset \beta$.

In order to interpret the $\mathbf{N}$-integral as a $B$-integral put

$$
\begin{aligned}
\beta_{\xi, E}= & \{(t,[c, d]) ; t-\xi(t)<c<t<d<t+\xi(t) \text { with } c, d \in I \backslash E\} \\
& \cup\{(t,[a, d]) ; t-\xi(t)<a \leq t<d<t+\xi(t) \text { with } d \in I \backslash E\} \\
& \cup\{(t,[c, b]) ; t-\xi(t)<c<t \leq d<t+\xi(t) \text { with } c \in I \backslash E\}, \\
B= & \left\{\beta_{\xi, E} ; \xi: I \rightarrow(0, \infty), E \in \mathbf{N}\right\} .
\end{aligned}
$$

Then the set of partitions $\Delta=\left\{\left(t,\left[x_{i-1}, x_{i}\right]\right)\right\}$ of $I$ which are $(\xi, E)$-fine is just the set of partitions $\Delta$ of $I$ which fulfil $\Delta \subset \beta$ and $B$-integration is $\mathbf{N}$-integration. (If $\mathbf{S}$ fulfils (6.1)-(6.4), (6.6), (6.7) and if $B=\left\{\beta_{\gamma} ; \gamma\right.$ is an S-choice $\}$ then $\mathbf{S}$-integration is $B$-integration.) 


\section{References}

[A-A 65] I. Amemyia and T. Ando, On the class of functions integrable in certain generalized sense, J. Fac. Sci. Hokkaido Univ. 18 (1995), 128-140.

[Al 50] A. Alexiewicz, On sequences of operations (II), Studia Mathematica 11 (1950), 200-236.

[Bo 96] B. Bongiorno, Un nuovo integrale per il problema delle primitive, Le Matematiche 51 (1996), 299-313.

[Bo-Pf 92] B. Bongiorno and W. F. Pfeffer, A concept of absolute continuity and a Riemann type integral, Comment. Math. Univ. Carolinae 33 (1992), 189-196.

[Bo-Pi 91] B. Bongiorno and L. Di Piazza, Convergence theorems for generalized RiemannStieltjes integrals, Real Analysis Exchange 17 (1991-92), 339-361.

[B-P-P 00] B. Bongiorno, L. Di Piazza and D. Preiss, A constructive minimal integral which includes Lebesgue integrable functions and derivatives, J. London Math. Soc. (2) 62 (2000), 117-126.

[B-P-S 95] B. Bongiorno, L. Di Piazza and V. Skvortsov, A new full descriptive characterization of Denjoy-Perron integral, Real Analysis Exchange 21 (1995-96), 656-663.

[B-P-S 02] B. Bongiorno L. Di Piazza and V. A. Skvortsov, On dyadic integrals and some other integrals associated with local systems, J. Math. Anal. Appl. 271 (2002), 506-524.

[B-P-S 03] B. Bongiorno, L. Di Piazza and V. A. Skvortsov, The Ward property for a $\mathcal{P}$-adic basis and the $\mathcal{P}$-adic integral, J. Math. Anal. Appl. 285 (2003), 578-592.

[Br-Kr 03] M. Brokate and P. Krejčí, Duality in the space of regulated functions and the play operator, Math. Z. 245 (2003), 667-688.

[Ch-D 78] V. G. Chelidze and A. G. Djvarsheisvili, The Theory of the Denjoy Integral and Some of its Applications, Tbilisi, 1978 (in Russian); in English, World Scientific, Singapore, 1989.

[D 51] A. G. Djvarsheisvili, On a sequence of integrals in the sense of Denjoy, Akad. Nauk Gruzin. SSR Trudy Mat. Inst. 18 (1951), 221-236 (in Russian).

[E 95] V. Ene, Real Functions-Current Topics, Lecture Notes in Math. 1603, SpringerVerlag, 1995.

[G 91] R. A. Gordon, A general convergence theorem for non-absolute integrals, J. London Math. Soc. 44 (1991), 301-309.

[G 92] R. A. Gordon, On the equivalence of two convergence theorems for the Henstock integral, Real Analysis Exchange 18 (1992-93), 261-266.

[He 61] R. Henstock, Definitions of Riemann type of the variational integrals, Proc. London Math. Soc. 11 (1961), 402-418.

[He 63] R. Henstock, Theory of Integration, Butterworths, London, 1963.

[Ho 75] Ch. S. Hönig, Volterra-Stieltjes Integral Equations, North-Holland Mathematics Studies 16 (1975).

[J-K-S 83] J. Jarník, J. Kurzweil and St. Schwabik, On Mawhin's approach to multiple nonabsolutely convergent integral, Čas. Pěst. Mat. 108 (1983), 356-380.

[Kr 03] P. Krejčí, The Kurzweil integral with exclusion of negligible sets, Math. Bohem. 128 (2003), 277-292.

[Kr-Kur 02] P. Krejčí and J. Kurzweil, A nonexistence result for the Kurzweil integral, Math. Bohem. 127 (2002), 571-580.

[Kr-L 02] P. Krejčí and P. Laurençot, Generalized variational inequalities, Journal of Convex Analysis 9 (2002), 159-183. 
[Kun 54] K. Kunugi, Sur les espaces complets et régulièrement complets, I, II, Proc. Japan Acad. 30 (1954), 553-556, 912-916.

[Kun 56] K. Kunugi, Application de la méthode des espaces rangés à la théorie de l'intégration I, Proc. Japan Acad. 32 (1956), 215-220.

[Kur 57] J. Kurzweil, Generalized ordinary differential equations and continuous dependence on a parameter, Czechoslovak Math. J. 7 (1957), 418-449.

[Kur 58a] J. Kurzweil, Generalized ordinary differential equations, Czechoslovak Math. J. 8 (1958), 360-388.

[Kur 58b] J. Kurzweil, Unicity of solutions of generalized differential equations, Czechoslovak Math. J. 8 (1958), 348-359.

[Kur 80] J. Kurzweil, Nichtabsolut konvergente Integrale, Teubner, Leipzig, 1980.

[Kur 00] J. Kurzweil, Henstock-Kurzweil Integration: Its Relation to Topological Vector Spaces, World Scientific, Singapore, 2000.

[Kur 02] J. Kurzweil, Integration between the Lebesgue Integral and the Henstock-Kurzweil Integral: Its Relation to Local Convex Vector Spaces, World Scientific, Singapore, 2002.

[Kur] J. Kurzweil, On Riemannian approach to integration, submitted to Real Anal. Exchange.

[Kur-J 87] J. Kurzweil and J. Jarník, Limit processes in ordinary differential equations, Zeitschrift für Angewandte Mathematik und Physik 38 (1987), 241-256.

[Kur-J 88] J. Kurzweil and J. Jarník, Iterated Lie brackets in limit processes in ordinary differential equations, Resultate der Mathematik 14 (1988), 125-137.

[Kur-J 96] J. Kurzweil and J. Jarník, Perron type integration on n-dimensional intervals as an extension of integration of stepfunctions by strong equiconvergence, Czechoslovak Math. J. 46 (1996), 1-20.

[L 89] P. Y. Lee, Lanzhou Lectures on Henstock Integration, World Scientific, Singapore, 1989.

[Ma 81] J. Mawhin, Generalized Perron integral and the Green-Goursat theorem for differentiable vector fields, Czechoslovak Math. J. 31 (1981), 614-632.

[Mc Sh 69] E. J. Mc Shane, A Riemann-type integral that includes Lebesgue-Stieltjes, Bochner and stochastic integrals, Mem. AMS 88 (1969).

[N 78,79] S. Nakanishi, Completions of ranked spaces I, II, Math. Japon. $23(1978,1979)$, 409-422, 629-646.

[N 90] S. Nakanishi, Methods of ranked spaces and the Kunugi integral, Southeast Asian Bull. Mathematics 14 (1990), 1-15.

[N 01] S. Nakanishi, Approach to non-absolute integration by successive approximations, Scientiae Mathematicae Japonicae 54 (2001), 391-426.

[O 54] U. S. Očan, Generalized integrals, Mat. Sbornik 28 (1951), 293-336.

[Pf 93] W. F. Pfeffer, The Riemann Approach to Integration, Cambridge University Press, 1993.

[Pf 99] W. F. Pfeffer, The Lebesgue and Denjoy-Perron integrals from a descriptive point of view, Ric. Mat. 48 (1999), 211-223.

[Sa 37] S. Saks, Theory of the Integral, G. E. Stechert, New York, 1937.

[Sch 92a] Št. Schwabik, Generalized Ordinary Differential Equations, World Scientific, Singapore, 1992.

[Sch 92b] Št. Schwabik, Linear operators in the spaces of regulated functions, Math. Bohem. 117 (1992), 79-92. 
[Sch-Tv 74] Št. Schwabik and M. Tvrdý, Boundary value problems for generalized linear differential equations, Czechoslovak Math. J. 29 (1974), 451-477.

[Ti 29] E. C. Titchmarsh, On conjugate functions, Proc. London Math. Soc. 29 (1929), 49-80.

[Th 90] B. S. Thomson, Real Functions, Lecture Notes in Math. 1170, Springer-Verlag, 1980 .

[Th $82 / 83 \mathrm{a}]$ B. S. Thomson, Derivation bases on the real line, Real Anal. Exchange 8 (1982/83), 67-207.

[Th $82 / 83 \mathrm{~b}$ ] B. S. Thomson, Derivation bases on the real line, Real Anal. Exchange 8 (1982/83), 278-442.

[Tv 73] M. Tvrdý, Boundary value problems for linear generalized differential equations and their adjoints, Czechoslovak Math. J. 23 (1973), 183-217.

[Tv 74] M. Tvrdý, Boundary value problems for generalized linear integrodifferential equations with left-continuous solutions, Časopis Pěst. Mat. 99 (1974), 147-157.

[Tv 91] M. Tvrdý, Generalized differential equations in the space of regulated functions (Boundary value problems and controllability), Math. Bohem. 116 (1991), 225244 .

[Tv 96] M. Tvrdý, Linear bounded functionals on the space of regular regulated functions, Tatra Mt. Math. Publ. 8 (1996), 203-210.

[Tv 98] M. Tvrdý, Linear integral equations in the space of regulated functions, Math. Bohem. 123 (1998), 177-212.

[Tv 99] M. Tvrdý, On the continuous dependence on a parameter of solutions of initial value problems for linear generalized differential equations, Funct. Differ. Equ. 5 (1999), 483-498.

[Tv 00] M. Tvrdý, Linear boundary value problems for generalized differential equations, Ann. Math. Sil. 14 (2000), 51-80.

[U 56] P. R. Ulyanov, On the A-integral of Cauchy, Uspehi Mat. Nauk 11 (1956), 19771980.

[W 61] A. Wiweger, Linear spaces with mixed topology, Studia Mathematica 20 (1961), 47-68. 